\title{
Role of CIP2A in the antitumor effect of bortezomib in colon cancer
}

\author{
YAYUN DING $^{1 *}$, YUEGUO WANG $^{2 *}$, SHAOQING JU $^{2}$, XINGHUA WU $^{4}$, \\ WENCAI ZHU ${ }^{2}$, FENG SHI ${ }^{1}$ and LIPING MAO ${ }^{3}$ \\ ${ }^{1}$ Department of Laboratory Medicine, Nantong University, ${ }^{2}$ Laboratory Department, Affiliated Hospital of Nantong University, \\ ${ }^{3}$ Laboratory Department, Nantong Third People's Hospital, ${ }^{4}$ Surgical Comprehensive Laboratory, \\ Affiliated Hospital of Nantong University, Nantong, Jiangsu 226001, P.R. China
}

Received September 12, 2013; Accepted March 13, 2014

DOI: $10.3892 / \mathrm{mmr} .2014 .2173$

\begin{abstract}
Cancerous inhibitor of protein phosphatase 2A (CIP2A) has been identified as an oncoprotein that is able to promote the proliferation of cancer cells. The role of CIP2A in the anticancer activity of bortezomib in colon cancer remains to be elucidated. In the present study, the antitumor effect of bortezomib was investigated and the role of CIP2A in determining the effect on colon cancer cells was identified. In the present study, bortezomib demonstrated an antitumor effect, as observed by WST-1 assay and flow cytometry. In addition, the mRNA and protein level of CIP2A was inhibited in a dose-dependent manner by bortezomib with quantitative PCR (qPCR) and western blotting. Furthermore, the inhibition of CIP2A with small interfering RNA by treatment with bortezomib inhibited proliferation, increased apoptosis and attenuated the invasion of the cells. Finally, the in vivo data demonstrated that bortezomib was able to decrease the growth of tumors, and that CIP2A was downregulated in the LoVo tumors treated with bortezomib. Therefore, CIP2A was shown to be important in the bortezomib-induced inhibitory effect on colon cancer.
\end{abstract}

\section{Introduction}

Colon cancer, the third most common malignancy worldwide, with more than 1 million new cases and 500,000 mortalities each year (1), remains one of the most difficult tumors to treat. Common risk factors include obesity, smoking and a high lifetime intake of alcohol (2-4). However, patients with colon cancer are almost always diagnosed at the advanced stage and

Correspondence to: Professor Liping Mao, Laboratory Department, Nantong Third People's Hospital, 99 Middle Qingnian Road, Nantong, Jiangsu 226001, P.R. China

E-mail: maoliping70@163.com

${ }^{*}$ Contributed equally

Key words: cancerous inhibitor of protein phosphatase 2A, bortezomib, colon cancer thus are not eligible for curative therapies. The high mortality and recurrence rates demonstrate the aggressiveness of this cancer. The development of new efficient treatments for colon carcinoma are therefore urgently required.

Bortezomib is the first proteasome inhibitor to be approved for treating patients with multiple myeloma, lymphoma and other types of hematological malignancy (5-7). Among the known targets of bortezomib in numerous types of cancer, nuclear factor $-\kappa \mathrm{B}(\mathrm{NF}-\kappa \mathrm{B})$, intracellular $\mathrm{NAD}^{+}$levels and heat shock proteins have become the most analyzed targets of bortezomib in previous studies (8-12). Furthermore, more and more studies have indicated that cancerous inhibitor of protein phosphatase 2A (CIP2A) is involved in bortezomib-mediated apoptosis (13-16). CIP2A, originally named KIAA1524 or P90, has been identified as an oncoprotein that is able to lead to the proliferation of cancer cells. In previous studies, CIP2A was overexpressed in certain types of tumor, including head and neck, gastric, colon and breast cancers (17-20). Previous studies (13-16) indicated that CIP2A was the major molecular target in bortezomib-induced apoptosis in head and neck squamous cell carcinoma (HNSCC), triple negative breast cancer (TNBC), leukemia cells and hepatocellular carcinoma (HCC).

However, the role of CIP2A in the antitumor effect of bortezomib in colon cancer remains unclear. In the present study, the antitumor activity of bortezomib in different colon tumor cell lines and the role of CIP2A in the treatment with bortezomib in colon cancer were investigated.

\section{Materials and methods}

Cell lines and cultures. Three human colon cancer cell lines, LoVo, SW620 and HCT116, were obtained from the Shanghai Anorectal Institute (Shanghai, China). All the colon cancer cell lines were cultured in Dulbecco's modified Eagle's medium (DMEM; Gibco-BRL, Rockville, MD, USA) supplemented with $10 \%$ fetal bovine serum (FBS; Gibco-BRL), $100 \mathrm{U} / \mathrm{ml}$ penicillin $\mathrm{G}$ and $2 \mathrm{mM}$ L-glutamine in a humidified, $5 \% \mathrm{CO}_{2}$ atmosphere at $37^{\circ} \mathrm{C}$. Bortezomib was purchased from Millennium Pharmaceuticals (Cambridge, MA, USA). The stock solutions were prepared in dimethyl sulfoxide (Gibco-BRL) and stored at $-80^{\circ} \mathrm{C}$ until use. 
Cell viability analysis. The effect of bortezomib on colon cancer cell viability was determined by a WST-1 Cell Cytotoxicity Assay kit (Roche Diagnostics, Karlsruhe, Germany) in 5 to 10 replicates. The cells were seeded and incubated in 96-well plates $\left(8.0 \times 10^{3}\right.$ cells $/ 100 \mu \mathrm{l} /$ well) for $24 \mathrm{~h}$, which were exposed to various concentrations of bortezomib $(0,40,80$ and $160 \mathrm{nM})$ for $48 \mathrm{~h}$. The controls received WST-1 in 10\% FBS-containing DMEM. Next, $10 \mu \mathrm{l}$ WST-1 was added into each well and incubated at $37^{\circ} \mathrm{C}$ for $2 \mathrm{~h}$. The optical density (OD) at $450 \mathrm{~nm}$ and $630 \mathrm{~nm}$ was measured by a Benchmark microplate reader (Bio-Tek Elx800; Bio-Tek Instruments, Inc., Winooski, VT, USA). The data were obtained from three independent experiments.

Apoptosis analysis. Bortezomib-induced apoptotic cell death was measured by flow cytometry using the Annexin-V-FLUOS Staining kit (Roche Diagnostics), according to the manufacturer's instructions.

RNA isolation and quantitative PCR ( $q P C R)$. Total RNA was isolated from cultured cells by TRIzol reagent (Invitrogen Life Technologies, Karlsruhe,Germany). The concentration and purity of RNA were determined by the $\mathrm{OD}_{260} / \mathrm{OD}_{280}$ ratio. The cDNA synthesis was performed using the cDNA Synthesis kit (Roche, Basel, Switzerland), according to the manufacturer's instructions. qPCR was performed using the following specific primers: CIP2A forward, 5'-CAAATCACAGTTTTCCAACATCA-3' and reverse, 5'-TCCTTTACCACCATTCCAGAC-3'); and glyceraldehyde-3-phosphate-dehydrogenase (GAPDH) forward, 5'-TCGGAGTCAACGGATTTGGTCGT-3' and reverse, 5'-TGCCATGGGTGGAATCATATTGGA-3'. GAPDH was selected as an internal control. The conditions were $95^{\circ} \mathrm{C}$ for $30 \mathrm{sec}, 60^{\circ} \mathrm{C}$ for $30 \mathrm{sec}$ and $72^{\circ} \mathrm{C}$ for $31 \mathrm{sec}$ for 35 cycles. qPCR was performed with an ABI PRISM 7500 Sequence Detection System using the SYBR-Green I master kit (Roche Diagnostics). The results were normalized to the mRNA level of GAPDH.

Western blot analysis. The lysates of the LoVo cells treated with bortezomib at different concentrations for $48 \mathrm{~h}$ were prepared for the immunoblotting of CIP2A. The cells were suspended in lysis buffer containing $1 \mathrm{mM}$ phosphatase inhibitor and $1 \mathrm{mM}$ phenylmethylsulfonyl fluoride. The lysates were centrifuged at $16,060 \mathrm{x} \mathrm{g}$ at $4^{\circ} \mathrm{C}$ for $20 \mathrm{~min}$. Total protein $(20 \mu \mathrm{g})$ extracts were separated by $10 \%$ SDS-PAGE and transferred onto polyvinylidene difluoride membranes. The membranes were inhibited with 5\% skimmed milk in Tris-buffered saline and Tween 20, and then incubated with rabbit polyclonal CIP2A (Bethyl Laboratories, Montgomery, TX, USA) and rabbit polyclonal anti- $\beta$-actin (Bioworld Technology, Inc., St. Louis Park, MN, USA) antibodies. The immunoblots were scanned by ChemiDoc XRS (Bio-Rad, Hercules, CA, USA).

Gene-knockdown using small interfering (si)RNA. The siRNA reagents, including control CIP2A, were all purchased from Invitrogen Life Technologies. The siRNA-CIP2A sequence was as follows: forward, 5'-CCUACUGAUGGAUCUCCUUTT-3' and reverse, 5'-AAGGAGAUCCAUCAGUAGGTT-3'. The LoVo cells were plated in 6 -well plates at $5 \times 10^{5}$ per well in $2 \mathrm{ml}$ DMEM with $10 \%$ FBS. Briefly, the LoVo cells were transfected with siRNA (final concentration of $200 \mathrm{pM}$ ) in 6-well plates using Lipofectamine 2000 transfection reagent (Invitrogen Life Technologies), according to the manufacturer's instructions. The colon cancer cells were incubated with bortezomib, harvested and separated for relative qPCR and western blot analysis.

Cell invasion assay. The cell invasion assay was performed in a 24-well Transwell chamber with a pore size of $8 \mu \mathrm{m}$ (Costar, New York, NY, USA). The inserts were coated with $30 \mu 1$ Matrigel (1:2 dilution; BD Biosciences, Franklin Lakes, NJ, USA), then $1 \times 10^{6}$ LoVo cells in $100 \mu \mathrm{l}$ serum-free medium were added to the upper Matrigel chamber and incubated for $48 \mathrm{~h}$. The number of invaded cells were counted in eight random fields (magnification, x100).

Xenograft tumor growth. Eight-week-old male nude mice were purchased from the Shanghai Laboratory Animal Center of the Chinese Academy of Sciences (Shanghai, China). The mice were maintained in a specific pathogen-free environment. All experimental procedures were performed in accordance with protocols that were approved by the Committee for the Care and Use of Laboratory animals, Shanghai Laboratory Animal Center of the Chinese Academy of Sciences. Each mouse was inoculated in the dorsal flank with $10^{6}$ LoVo cells suspended in $100 \mu \mathrm{l}$ DMEM containing 50\% Matrigel. When the tumors reached a volume of $100 \mathrm{~mm}^{3}$, the mice were injected ( $1 \mathrm{mg} / \mathrm{kg}$ body weight) twice weekly and the controls received the vehicle. Calipers were used to measure the tumor volume weekly. The tumor volumes were calculated according to the following formula: width ${ }^{2} \mathrm{x}$ length $\mathrm{x} 0.52$ (21).

Statistical analysis. Quantitative data are expressed as the mean \pm standard error of the mean, which was obtained from three separate experiments. The statistical comparisons were based on t-test analysis. $\mathrm{P}<0.05$ was considered to indicate a statistically significant difference. All statistical analyses were performed using SPSS software (Windows version 17.0; SPSS, Inc., Chicago, IL, USA).

\section{Results}

Effect of bortezomib on viability and apoptosis in colon cancer cells. In order to identify the antitumor effect of bortezomib on colon cancer cells, the inhibitory effect of bortezomib was assessed in LoVo, SW620 and HCT116 colon cancer cell lines at different concentrations. The cell viability was determined by a WST-1 assay following $48 \mathrm{~h}$ of treatment. As shown in Fig. 1A, bortezomib exhibited similar effects on the viability of the three types of cells. Bortezomib caused a significant reduction in the colon cell viability in the $\mathrm{LoVo}\left(\mathrm{IC}_{50}, 39 \mathrm{nmol} / \mathrm{l}\right)$, SW620 ( $\left.\mathrm{IC}_{50}, 79 \mathrm{nmol} / \mathrm{l}\right)$ and HCT116 ( $\left.\mathrm{IC}_{50}, 81 \mathrm{nmol} / \mathrm{l}\right)$ cells.

Evidence shows that the inhibition of growth is caused by the induction of apoptosis (22). Apoptosis was determined by flow cytometry in the LoVo cells. As shown in Fig. 1B, bortezomib caused a clear increase in cell apoptosis, with an apoptotic rate of $1.9,8.7,15.8$ and $41.2 \%$ at concentrations of 0 , 40, 80 and $160 \mathrm{nmol} / 1$, respectively.

Bortezomib inhibits the transcription of CIP2A in colon cancer cells. Previous studies have demonstrated that CIP2A 
A

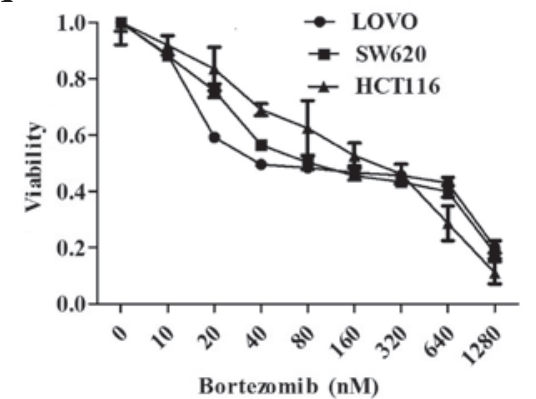

B

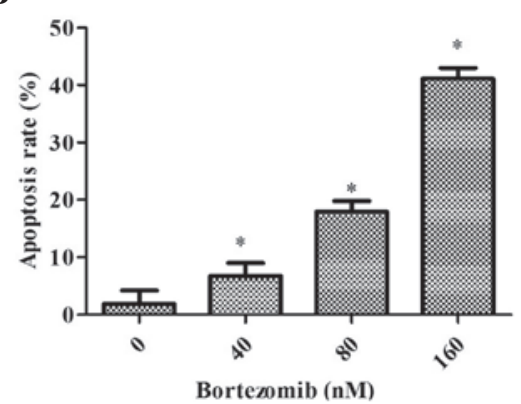

Figure 1. Bortezomib-mediated antiproliferation and proapoptosis in colon cancer cells. (A) Cell viability was examined using a WST-1 assay in cells treated for $48 \mathrm{~h}$ with the indicated concentrations of bortezomib. (B) Apoptosis of cells treated at the indicated doses of bortezomib for $48 \mathrm{~h}$ was determined by flow cytometry. Data are representative of three independent experiments, ${ }^{*} \mathrm{P}<0.05$ vs. vehicle.

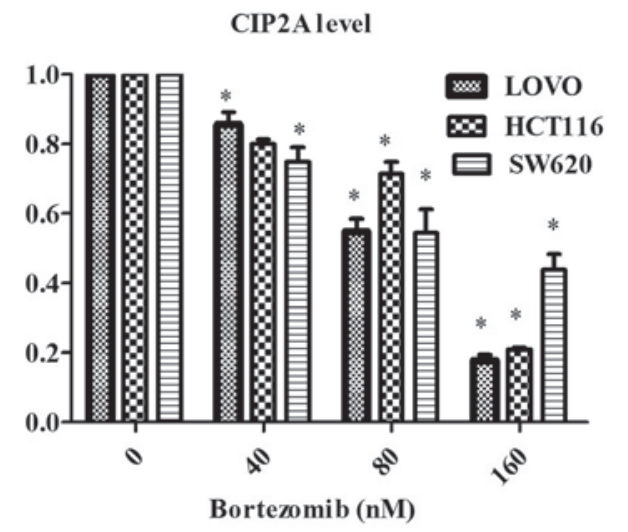

Figure 2. Bortezomib inhibits the transcription of CIP2A in colon cancer cells. The transcription of CIP2A was performed by qPCR. The colon cancer cell lines were exposed to bortezomib at the indicated doses for $48 \mathrm{~h}$. Data are representative of three independent experiments, ${ }^{*} \mathrm{P}<0.05$ vs. vehicle. CIP2A, cancerous inhibitor of protein phosphatase $2 \mathrm{~A}$; qPCR, quantitative PCR.

mediates the bortezomib-induced inhibitory effect on TNBC, HNSCC, leukemia cells and HCC (13-16). The present study assessed whether CIP2A plays a significant role in the bortezomib-induced antitumor effect. Thus, whether bortezomib affected the transcription of CIP2A was examined. qPCR indicated that bortezomib inhibited the mRNA level of CIP2A in a dose-dependent manner (Fig. 2)

Bortezomib downregulates the protein level of CIP2A in LoVo cells. Next, it was investigated whether the decreased mRNA level of CIP2A lead to a decreased protein level of CIP2A. Therefore, the protein level of CIP2A was examined in the LoVo cells treated with bortezomib. Whole cell extracts of the LoVo cells treated as described previously were subjected to western blotting with the CIP2A antibody. As shown in Fig. 3, the protein expression was inhibited in a dose-dependent manner. This result was consistent with the mRNA level of CIP2A.

Knockdown of CIP2A increases the antitumor effect of bortezomib on colon cancer cells. Since CIP2A appeared to be markedly inhibited by bortezomib, the following experiments were performed to validate the role of CIP2A signaling in the bortezomib-induced inhibitory effect on colon cancer cell lines, which was responsible for the effect in vitro. The protein expression of CIP2A was knocked down in the LoVo
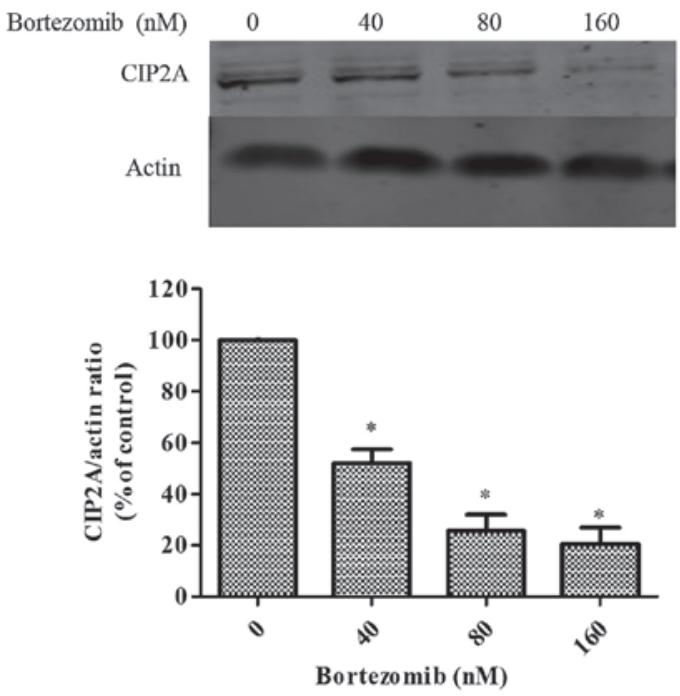

Figure 3. Bortezomib downregulates the protein level of CIP2A in LoVo cells. The cells were treated with the indicated concentrations of bortezomib for $48 \mathrm{~h}$. The protein level of CIP2A was evaluated by western blotting. Data are representative of three independent experiments, ${ }^{*} \mathrm{P}<0.05$ vs. vehicle. CIP2A, cancerous inhibitor of protein phosphatase $2 \mathrm{~A}$.

cell line using siRNA. Notably, proliferation differences between scrambled siRNA and CIP2A siRNA were observed (Fig. 4A). The $\mathrm{IC}_{50}$ values of scrambled siRNA and CIP2A siRNA were 170 and $90 \mathrm{nmol} / 1$, respectively. Additionally, the downregulation of CIP2A sensitized the LoVo cell line and increased the apoptotic rate from 15.8 to $31.2 \%$ at a concentration of $80 \mathrm{nmol} / \mathrm{l}$ (Fig. 4B). Furthermore, the LoVo cells were transfected with scrambled siRNA and CIP2A siRNA for $48 \mathrm{~h}$. The invasion capability of the LoVo cells transfected with CIP2A siRNA was significantly inhibited at $48 \mathrm{~h}$ (LoVo control vs. CIP2A siRNA: $120 \pm 21$ vs. $48 \pm 6$ cells; Fig. 4C).

Effect of bortezomib on colon cancer cell xenograft tumors. To confirm whether the antitumor effect of bortezomib on CIP2A has clinical implications, the effect of bortezomib on xenograft tumors in vivo was examined. Tumor-bearing mice were exposed to bortezomib and the vehicle at $1.0 \mathrm{mg} / \mathrm{kg}$ twice a week. No pathological abnormalities were observed at necropsy. The data (Fig. 5A) indicated that treatment with bortezomib twice a week had a marked inhibitory effect on the LoVo tumors ( $\left.{ }^{*} \mathrm{P}<0.05\right)$, and the tumor size in the treatment 
A

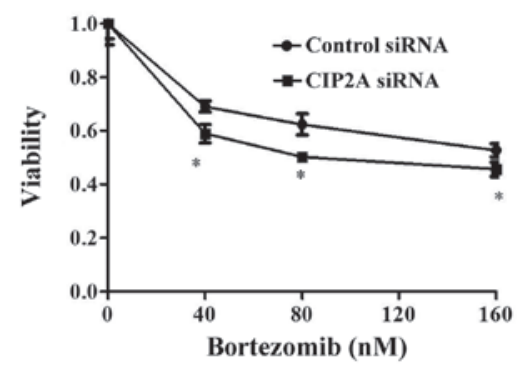

B
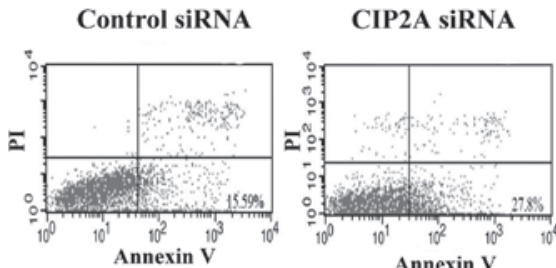

C
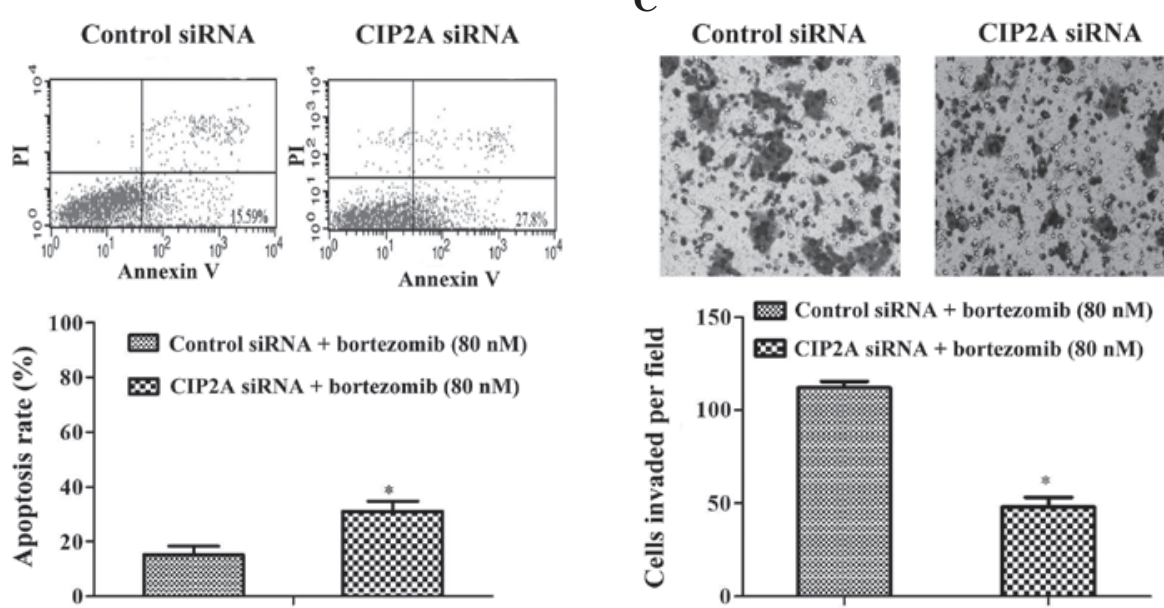

Figure 4. Target validation of CIP2A. (A) Cells were transfected with either CIP2A siRNA or the control for $48 \mathrm{~h}$, and then treated with bortezomib for $48 \mathrm{~h}$. Apoptosis was determined by flow cytometry at the indicated doses. A statistically significant difference was observed ("P<0.05). (B) The numbers of LoVo cells invading the lower surface of the filter were counted following treatment with control siRNA and CIP2A siRNA. A statistically significant difference was observed ("P<0.05). (C) A WST-1 assay was performed following treatment with siRNA and bortezomib. The absorbance was reduced. Each of the experiments were repeated in triplicate. CIP2A, cancerous inhibitor of protein phosphatase 2A; siRNA, small interfering RNA.

A

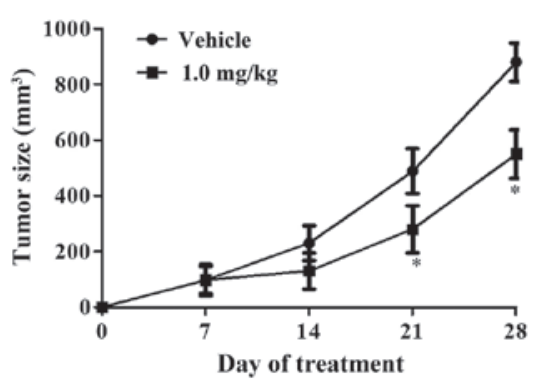

B

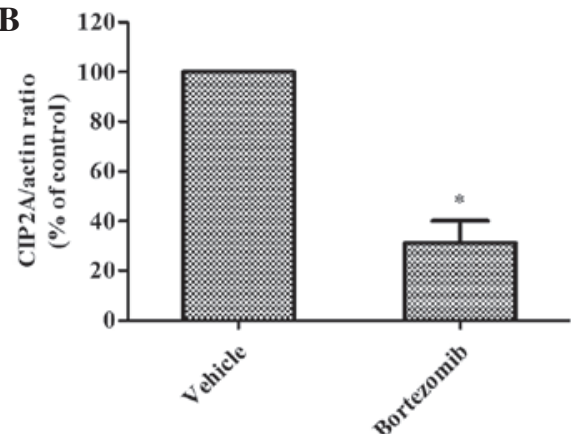

Figure 5. In vivo effect of bortezomib on colon cancer cell lines in xenograft nude mice. (A) Bortezomib was able to decrease the size of the tumors. ${ }^{*} \mathrm{P}<0.05$ vs. vehicle. (B) CIP2A expression in the tumors was examined by western blot analysis. The mice were exposed to bortezomib (1 mg/kg body weight) twice weekly. The control received the vehicle. ${ }^{\mathrm{P}}<0.05$ vs. vehicle. CIP2A, cancerous inhibitor of protein phosphatase $2 \mathrm{~A}$.

group was just $40 \%$ of that in the control group. Furthermore, to investigate the mechanisms underlying the biological response, CIP2A was examined in the LoVo cells by western blotting (Fig. 5B). Overall, there was a clear decrease in CIP2A in the LoVo cells treated with bortezomib. These results were in line with the data of the LoVo cells treated in vitro.

\section{Discussion}

CIP2A has been identified as an oncoprotein that is able to stabilize the expression of the c-Myc protein. In addition, it is able to promote anchorage-independent cell growth and tumor formation in vivo. Furthermore, elevated expression of CIP2A has been investigated in numerous types of human malignancy, including prostate, gastric, bladder, ovarian and non-small cell lung cancers, hepatocellular carcinoma, acute myeloid leukemia and astrocytomas (16,22,24-29). Furthermore, with respect to colon cancer, CIP2A is overexpressed in colon cancer tissue compared with the normal colon mucosa. In addition, the knockdown of CIP2A has been shown to reduce proliferation and anchorage-independent colony formation (30). Liu et al indicated that CIP2A was 
important in numerous carcinomas and served as a novel therapeutic target (15). Thus, the investigation of therapeutic CIP2A-targeted treatment is necessary. However, the function of CIP2A in the bortezomib-induced inhibitory effect in colon cancer cell lines is unclear. Therefore, the present study investigated the antitumor effect of bortezomib and identified the role of CIP2A that determined the antitumor action in colon cancer cells.

In the present study, bortezomib inhibited colon cancer cells in vitro and in vivo (Figs. 1 and $5 \mathrm{~A}$ ). This result was in line with a previous study that indicated that bortezomib caused a dose-dependent reduction of cell viability and an increase of apoptosis in colon cancer cells (31).

Furthermore, the mechanisms underlying the effect of bortezomib require further investigation. The present findings clearly demonstrated that the treatment of bortezomib was able to downregulate the mRNA and protein levels of CIP2A in a dose-dependent manner (Figs. 2 and 3). It has been reported that c-Myc has a key role in colon cancer (30). Additionally, CIP2A has been demonstrated to promote the phosphorylation of myc serine 62 and thereby prevent the proteolytic degradation of myc in numerous carcinoma cells (17). Therefore, colon cancer growth may depend on the association between CIP2A and myc. Furthermore, the present findings clearly demonstrated that bortezomib treatment was able to downregulate the mRNA and protein levels of CIP2A in a dose-dependent manner (Figs. 2 and 3). Thus, the anti-tumor effect of bortezomib may depend on the decreased expression of myc and CIP2A.

Furthermore, there are certain important indications that can be taken from the present study. CIP2A is important in the antitumor effect of bortezomib, which demonstrates that the antitumor effect of bortezomib does not necessarily rely on proteasome downregulation. Furthermore, the downregulation of CIP2A by siRNA was able to increase the efficiency of the antitumor activity of bortezomib (Fig. 4), whereas the colon cancer cells with a high expression of CIP2A demonstrated less activity (Fig. 4). The data were in line with previous studies that demonstrated that the downregulation of CIP2A was able to increase the efficacy of 5-fluorouracil, SN38 and oxaliplatin in colon cell lines (30). However, the high expression of CIP2A has also been shown to increase the drug resistance to doxorubicin in breast cancer (32). In addition, the results indicate that CIP2A may be a mediator of the antitumor effects of bortezomib rather than a bystander effect. Furthermore, these findings may imply that colon cancer cells with a high level of CIP2A expression may be more resistant to bortezomib. By contrast, the drugs that are able to clearly inhibit CIP2A expression may demonstrate significant sensitivity to the colon cancer cells.

At present, treating patients with molecular-targeted therapy is a popular approach. The present study discloses the fact that targeting CIP2A is able to improve the efficiency of bortezomib in colon cancer cell lines. This may offer a meaningful policy for clinical therapy in patients with colon cancer. However, despite the current findings, the manner in which bortezomib downregulates CIP2A remains to be elucidated and further mechanistic investigations are therefore necessary.

In summary, the present study demonstrated the antitumor activity of bortezomib and showed that the functional loss of
CIP2A occurred through the action of bortezomib. In addition, the downregulation of CIP2A increased the antitumor efficiency of bortezomib. Finally, the in vivo data demonstrated that bortezomib was able to decrease the growth of tumors and that CIP2A was downregulated in the LoVo tumors treated with bortezomib. Taken together, the results of the present study indicated that CIP2A is important in the antitumor effect of bortezomib in colon cancer. The present study also demonstrated that oncoproteins may be a potential antitumor treatment.

\section{Acknowledgements}

This study was supported by grants from the Health Innovation Teams of Jiangsu province and was performed in the Surgical Comprehensive Laboratory, Affiliated Hospital of Nantong University and Nantong University of Medicine, Jiangsu, China.

\section{References}

1. Naishadham D, Lansdorp-Vogelaar I, Siegel R, Cokkinides V and Jemal A: State disparities in colorectal cancer mortality patterns in the United States. Cancer Epidemiol Biomarkers Prev 20: 1296-1302, 2011.

2. Jemal A, Bray F, Center MM, Ferlay J, Ward E and Forman D: Global cancer statistics. CA Cancer J clin 61: 69-90, 2011.

3. Center MM, Jemal A and Ward E: International trends in colorectal cancer incidence rates. Cancer Epidemiol Biomarkers Prev 18: 1688-1694, 2009.

4. Ferrari P, Jenab M, Norat T, et al: Lifetime and baseline alcohol intake and risk of colon and rectal cancers in the European prospective investigation into cancer and nutrition (EPIC). Int J Cancer 121: 2065-2072, 2007.

5. Adams J and Kauffman M: Development of the proteasome inhibitor Velcade (Bortezomib). Cancer Invest 22: 304-311, 2004.

6. Goy A, Younes A, McLaughlin P, et al: Phase II study of proteasome inhibitor bortezomib in relapsed or refractory B-cell non-Hodgkin's lymphoma. J Clin Oncol 23: 667-675, 2005.

7. O'Connor OA, Wright J, Moskowitz C, et al: Phase II clinical experience with the novel proteasome inhibitor Bortezomib in patients with indolent non-Hodgkin's lymphoma and mantle cell lymphoma. J Clin Oncol 23: 676-684, 2005.

8. Orlowski RZ and Baldwin AS Jr: NF-kappaB as a therapeutic target in cancer. Trends Mol Med 8: 385-389, 2002.

9. Orlowski RZ and Kuhn DJ: Proteasome inhibitors in cancer therapy: lessons from the first decade. Clin Cancer Res 14: 1649-1657, 2008

10. Voorhees PM and Orlowski RZ: The proteasome and proteasome inhibitors in cancer therapy. Annu Rev Pharmacol Toxicol 46: 189-213, 2006.

11. QI W, White MC, Choi W, Guo C, Dinney C, McConkey DJ and Siefker-Radtke A: Inhibition of inducible heat shock protein-70 (hsp72) enhances bortezomib-induced cell death in human bladder cancer cells. PLoS One 8: e69509, 2013.

12. Cagnetta $\mathrm{A}, \mathrm{Cea} \mathrm{M}$, Calimeri $\mathrm{T}$, et al: Intracellular $\mathrm{NAD}^{+}$ depletion enhances bortezomib-induced anti-myeloma activity. Blood 122: 1243-1255, 2013.

13. Tseng LM, Liu CY, Chan KC, Chu PY, Shiau CW and Chen KF: CIP2A is a target of Bortezomib in human triple negative breast cancer cells. Breast Cancer Res 14: R68, 2012.

14. Lin YC, Chen KC, Chen CC, Cheng AL and Chen KF: CIP2A-mediated Akt activation plays a role in bortezomib-induced apoptosis in head and neck squamous cell carcinoma cells. Oral Oncol 48: 585-593, 2012.

15. Liu CY, Shiau CW, Kuo HY, Huang HP, Chen MH, Tzeng CH and Chen KF: Cancerous inhibitor of protein phosphatase 2A determines bortezomib-induced apoptosis in leukemia cells. Haematologica 98: 729-738, 2013.

16. Chen KF, Liu CY, Lin YC, Yu HC, Liu TH, Hou DR, Chen PJ and Cheng AL: CIP2A mediates effect of bortezomib on phospho-Akt and apoptosis in hepatocellular carcinoma cells. Oncogene 29: 6257-6266, 2010. 
17. Junttila MR, Puustinen P, Niemelä M, et al: CIP2A inhibits PP2A in human malignancies. Cell 130: 51-62, 2007.

18. Li W, Ge Z, Liu C, Björkholm M, Jia J and Xu D: CIP2A is overexpressed in gastric cancer and its depletion leads to impaired clonogenicity, senescence, or differentiation of tumor cells. Clin Cancer Res 14: 3722-3728, 2008.

19. Böckelman C, Koskensalo S, Hagström J, Lundin M, Ristimäkil A and Haglund C: CIP2A overexpression is associated with c-Myc expression in colorectal cancer. Cancer Biol Ther 13: 289-295, 2012

20. Côme C, Laine A, Chanrion M, et al: CIP2A is associated with human breast cancer aggressivity. Clin Cancer Res 15: 5092-5100, 2009.

21. Kulp SK, Yang YT,Hung CC, et al: 3-phosphoinositide-dependent protein kinase-1/Akt signaling represents a major cyclooxygenase-2-independent target for celecoxib in prostate cancer cells. Cancer Res 64: 1444-1451, 2004.

22. Chen KF, Yeh PY, Yeh KH, et al: Down-regulation of phospho-Akt is a major molecular determinant of bortezomib-induced apoptosis in hepatocellular carcinoma cells. Cancer Res 68: 6698-6707, 2008.

23. Vaarala MH, Väisänen MR, Ristimäki A: CIP2A expression is increased in prostate cancer. J Exp Clin Cancer Res 29: 136, 2010.

24. Dong QZ, Wang Y, Dong XJ,Li ZX, Tang ZP, Cui QZ and Wang EH: CIP2A is overexpressed in non-small cell lung cancer and correlates with poor prognosis. Ann Surg Oncol 18: 857-865, 2011.
25. Coenen EA, Zwaan CM, Meyer C and et al: KIAA1524: a novel MLL translocation partner in acute myeloid leukemia. Leuk Res 35: 133-135, 2011.

26. Khanna A, Böckelman C, Hemmes A, et al: MYC-dependent regulation and prognostic, role of CIP2A in gastric cancer. J Natl Cancer Inst 101: 793-805, 2009.

27. Yi F, Ni W, Bai J and Li W: Expression and biological role of CIP2A in human astrocytoma. Mol Med Rep 7: 1376-1380, 2013.

28. Huang LP, Savoly D, Sidi AA, Adelson ME, Mordechai E and Trama JP: CIP2A protein expression in high-grade, high-stage bladder cancer. Cancer Med 1: 76-81, 2012.

29. Böckelman C, Lassus H, Hemmes A, et al: Prognostic role of CIP2A expression in serous ovarian cancer. Br J Cancer 105: 989-995, 2011.

30. Teng HW, Yang SH, Lin JK: CIP2A is a predictor of poor prognosis in colon cancer. J Gastrointest Surg 16: 1037-1047, 2012.

31. Pitts TM, Morrow M, Kaufman SA, Tentler JJ and Eckhardt SG: Vorinostat and bortezomib exert synergistic antiproliferative and proapoptotic effects in colon cancer cell models. Mol Cancer Ther 8: 342-349, 2009.

32. Choi YA, Park JS, Park MY, et al: Increase in CIP2A expression is associated with doxorubicin resistance. FEBS Lett 585: 755-760, 2011. 